\title{
FUNDAMENTAL FREQUENCIES OF BIDIRECTIONAL FUNCTIONALLY GRADED SANDWICH BEAMS PARTIALLY SUPPORTED BY FOUNDATION USING DIFFERENT BEAM THEORIES
}

\author{
Vu Thi An Ninh \\ University of Transport and Communications, No 3 Cau Giay Street, Hanoi, Vietnam \\ ARTICLE INFO \\ TYPE: Research Article \\ Received: $27 / 12 / 2020$ \\ Revised: 03/03/2021 \\ Accepted: 08/03/2021 \\ Published online: 27/05/2021 \\ https://doi.org/10.47869/tcsj.72.4.5 \\ * Corresponding author \\ Email: vuthianninh@utc.edu.vn
}

\begin{abstract}
Investigation on the influence of beam theory and partial foundation support on natural frequencies play an important role in design of structures. In this paper, fundamental frequencies of a bidirectional functionally graded sandwich (BFGSW) beam partially supported by an elastic foundation are evaluated using various beam theories. The core of the sandwich beam is homogeneous while its two face sheets are made from three distinct materials with material properties varying in both the length and thickness directions by power gradation laws. The finite element method is employed to derive equation of motion and to compute the frequencies of the beam. The effects of the material gradation, the foundation parameters and the span to height ratio on the frequencies are studied in detail and highlighted. The difference of the frequencies obtained by different beam theories is also examined and discussed. The numerical results of the paper are useful in designing BFGSW beams with desired fundamantal frequencies.
\end{abstract}

Keywords: BFGSW beam, partial foundation support, frequency, finite element method.

(C) 2021 University of Transport and Communications

\section{INTRODUCTION}

A functionally graded material (FGM), a special composite material initiated by Japanese scientists in 1984 [1], possesses a continuous variation of material properties in one or more directions. This material is recently employed in fabricating sandwich structures to overcome the drawbacks of conventional sandwich structures such as the delamination, stress concentration at the interface of the layers. With high rigidity, low specific weight and high 
thermal and impact resistance, functionally graded sandwich (FGSW) structures have wide applications in aerospace, nuclear reactor, civil fields. Many investigations on mechanical behaviour of FGSW structures have been reported in recent years, contributions that are most relevant to the present work are briefly discussed below.

Based on different beam models, Apetre et al. [2] studied bending behavior of FGSW beams with a FGM core using a combined Fourier and series-Galerkin methods. Free vibration of sandwich beam with FGM core was investigated by Amirani et al. [3] using the element free Galerkin method. The authors showed that the natural frequencies obtained from Mori-Tanaka scheme are lower than that calculated from Voigt model. Free vibration and buckling of FGSW beams were investigated by Vo et al. [4] using refined shear deformation theory. The authors showed that the material grading index, the layer thickness and aspect ratios, boundary conditions have a significant influence on the frequency parameters and buckling loads. Based on a quasi-3D shear deformation theory and the symmetric smoothed particle hydrodynamics method, Karamanli [5] investigated bending behaviour of FGSW beams with material properties varying in both the length and thickness directions by the power gradation laws. Free vibration of of the beam with various boundary conditions was studied by Ninh et al. [6] using a new BFGSW beam model made from three constituent materials. Using the beam model in [6], Nguyen et al. [7] examined dynamic behaviour of the beam with simply supported ends under different motions of moving load.

Beams on elastic foundation are widely seen in practice, especially in civil engineering. Understanding the behaviour of the beam resting on elastic foundation, therefore is important for design engineers. Many studies on the behaviour of beams resting on elastic foundation have been reported in the literature. Eisenberger et al. [8] derived the exact stiffness and mass matrices of a beam element for computing natural frequencies and mode shapes of the beam supported by a Winkler elastic foundation. The state space method was used in combination with the differential quadrature method by Chen et al. [9] in studying bending and free vibration of a beam on a Pasternak elastic foundation. Bending behaviour of a simply supported FGSW beam with elastic core resting on a Pasternak foundation was studied by Zenkour et al. [10] using a refined sinusoidal shear deformation beam theory. Timoshenko beam theory and Chebyshev collocation method were employed by Tossapanon and Wattanasakulpong [11] to examine buckling and free vibration of FGSW beam resting on a two-parameter foundation. Su et al. [12] analysed free vibration of FGSW beam resting on a Pasternak foundation using the general Fourier method. Both the Voigt model and MoriTanaka scheme were employed by the authors to estimate the effective material properties of the beam.

Various beam theories are proposed for analysis of FGM beams in recent years [13]. Regarding free vibration analysis of FGM beams, Aydogdu et al. [14] employed the firstorder, parabolic and exponential shear deformation beam theories in evaluating frequencies of simply supported FGM beams. The classical, first-order and the third-order shear deformation beam theories were used by Şimșek [15] in analysing vibration of FGM beams under moving 
mass. The author showed that the beam theories lead to some difference in the dynamic deflections. An analytical method based on various beam theories was presented by Mahi et al. [16] for determining frequencies of FGM beams in thermal environment. The influence of various higher-order shear deformation theories on the bending and frequencies of FGM beams was studied by Thai and Vo [17].

As seen from above literature review, the beam theory has an important role on frequencies of FGM beams, and this topic is explored in the present work in some more factors by evaluating fundamental frequencies of bidirectional functionally graded sandwich (BFGSW) beams partially supported by a Pasternak foundation. The beam is assumed to consist of three layers, a homogenous core, and two FGM face sheets. The FGM sheets is made of three distinct materials whose volume fraction varying in both the length and thickness directions by power gradation laws. The displacement components are written a generalized form to demonstrate beam theories such as classical beam theory (CBT), the firstorder shear deformation beam theory (FSDBT), parabolic shear deformation beam theory (PSDBT), trigonometric shear deformation beam theory (TSDBT), exponential shear deformation beam theory (ESDBT), hyperbolic shear deformation beam theory (HSDBT). The equations of motion are derived from the Hamilton's principle and solved by the finite element method. The effects of the material distribution, the layer thickness and aspect ratios, the foundation parameters and the beam theories on the frequency parameter of the BFGSW beam are examined and discussed.

\section{BFGSW BEAM}

Figure 1 shows a BFGSW beam with length $L$, rectangular cross section $(b \times h)$, partially supported by an elastic foundation. The beam consists of three layers, a homogenous core, and two FGM face layers. The foundation is modelled by a Pasternak foundation, represented by Winkler elastic springs with stiffness $k_{w}$ and a shear layer with stiffness $k_{s}$. In figure $1, \alpha_{F}$ is the ratio of the supported part $L_{F}$ to the total beam length $L$. The Cartesian coordinate system $(x, y, z)$ is chosen such that the $x$-axis is on the mid-plane, and the $z$-axis is perpendicular to the mid-plane. Denoting $z_{0}=-h / 2, z_{1}, z_{2}$ and $z_{3}=h / 2$ are, respectively, the vertical coordinates of the bottom surface, the interfaces between the layers and the top surface.
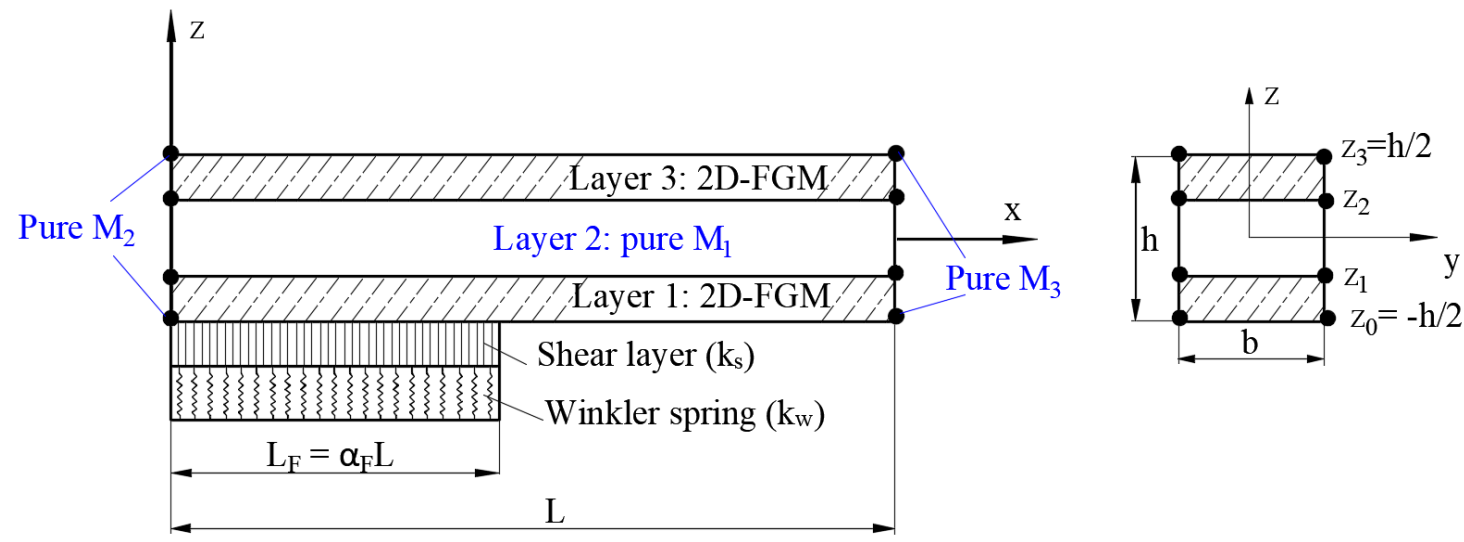

Figure 1. BFGSW beam partially supported by a Pasternak elastic foundation. 
Transport and Communications Science Journal, Vol. 72, Issue 4 (05/2021), 452-467

The beam is made from three distinct materials, $\mathrm{M}_{1}, \mathrm{M}_{2}$ and $\mathrm{M}_{3}$. The volume fractions of $\mathrm{M}_{1}, \mathrm{M}_{2}$ and $\mathrm{M}_{3}$ vary in both the $x$ and $z$ directions according to [7]

$$
\begin{aligned}
& \left\{\begin{array}{l}
V_{1}=\left(\frac{z-z_{0}}{z_{1}-z_{0}}\right)^{n_{z}} \\
V_{2}=\left[1-\left(\frac{z-z_{0}}{z_{1}-z_{0}}\right)^{n_{z}}\right]\left[1-\left(\frac{x}{L}\right)^{n_{x}}\right] \quad \text { for } z \in\left[z_{0}, z_{1}\right] \\
V_{3}=\left[1-\left(\frac{z-z_{0}}{z_{1}-z_{0}}\right)^{n_{z}}\right]\left(\frac{x}{L}\right)^{n_{x}} \\
V_{1}=1, V_{2}=V_{3}=0 \\
V_{1}=\left(\frac{z-z_{3}}{z_{2}-z_{3}}\right)^{n_{z}} \quad \text { for } z \in\left[z_{1}, z_{2}\right] \\
V_{2}=\left[1-\left(\frac{z-z_{3}}{z_{2}-z_{3}}\right)^{n_{z}}\right]\left[1-\left(\frac{x}{L}\right)^{n_{x}}\right] \\
V_{3}=\left[1-\left(\frac{z-z_{3}}{z_{2}-z_{3}}\right)^{n_{z}}\right]\left(\frac{x}{L}\right)^{n_{x}}
\end{array} \quad \text { for } z \in\left[z_{2}, z_{3}\right]\right.
\end{aligned}
$$

where $V_{1}, V_{2}$ and $V_{3}$ are, respectively, the volume fraction of $\mathrm{M}_{1}, \mathrm{M}_{2}$ and $\mathrm{M}_{3} ; n_{x}$ and $n_{z}$ are the axial and transverse grading indexes. The model defines a softcore sandwich beam if $\mathrm{M}_{1}$ is metal and a hardcore beam if $\mathrm{M}_{1}$ is a ceramic. Figure 2 shows the distribution in the longitudinal and thickness directions of $V_{1}, V_{2}$ and $V_{3}$ for $n_{x}=n_{z}=1, z_{1}=-h / 10, z_{2}=3 h / 10$.

The effective material properties, $P_{f}$, such as elastic modulus $E_{f}$, mass density $\rho_{f}$ evaluated by Voigt's model are of the form

$$
P_{f}(x, z)=P_{1} V_{1}+P_{2} V_{2}+P_{3} V_{3}
$$

where $P_{1}, P_{2}$ and $P_{3}$ are, respectively, the properties of $\mathrm{M}_{1}, \mathrm{M}_{2}$ and $\mathrm{M}_{3}$.
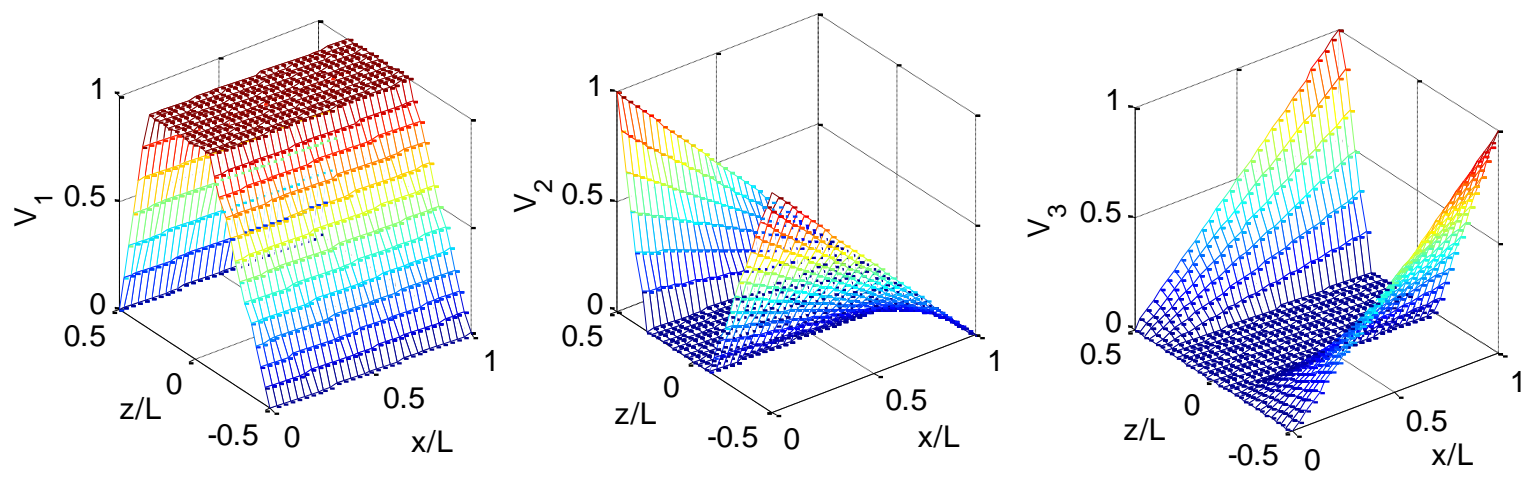

Figure 2. Distribution of $V_{1}, V_{2}$ and $V_{3}$ for $n_{x}=n_{z}=1, z_{1}=-h / 10, z_{2}=3 h / 10$.

Substituting eq. (1) into eq. (2), one gets 
Transport and Communications Science Journal, Vol. 72, Issue 4 (05/2021), 452-467

$$
P_{f}(x, z)= \begin{cases}{\left[P_{1}-P_{23}(x)\right]\left(\frac{z-z_{0}}{z_{1}-z_{0}}\right)^{n_{z}}+P_{23}(x)} & \text { for } z \in\left[z_{0}, z_{1}\right] \\ P_{1} & \text { for } z \in\left[z_{1}, z_{2}\right] \\ {\left[P_{1}-P_{23}(x)\right]\left(\frac{z-z_{3}}{z_{2}-z_{3}}\right)^{n_{z}}+P_{23}(x)} & \text { for } z \in\left[z_{2}, z_{3}\right]\end{cases}
$$

with $P_{23}(x)=P_{2}-\left(P_{2}-P_{3}\right)\left(\frac{x}{L}\right)^{n_{x}}$. One can easily verify that if $n_{x}=0$ or $\mathrm{M}_{2}$ is identical to $\mathrm{M}_{3}$, eq. (3) returns to the effective properties of the unidirectional transverse FGM sandwich beam in [12].

\section{MATHEMATICAL MODEL}

The displacements in the $x$ and $z$ directions of an arbitrary point in the beam, $u_{1}(x, z, t)$ and $u_{3}(x, z, t)$, are, respectively, written in the generalized form as follows

$$
u_{1}(x, z, t)=u(x, t)-g(z) w_{, x}(x, t)-f(z) \theta(x, t) ; \quad u_{3}(x, z, t)=w(x, t)
$$

where $u(x, t)$ and $w(x, t)$ are, respectively, the axial and transverse displacements of the point on the mid-plane; $\theta(x, t)$ is the rotation of the cross-section; $t$ is the time variable; a subscript comma is used to denote the derivative with respect to the variable which follows; the shape functions $g(z)$ and $f(z)$ are shown in Table 1 where different beam theories can be obtained by choosing $g(z)$ and $f(z)$.

Table 1. Shape functions $f(z)$ and $g(z)$ for different beam theories.

\begin{tabular}{lll}
\hline$g(z)$ & $f(z)$ & Theory \\
\hline$z$ & 0 & CBT \\
0 & $z$ & FSDBT \\
$4 z^{3} / 3 h^{2}$ & $z\left(4 z^{2} / 3 h^{2}-1\right)$ & PSDBT \\
$z$ & $-h / \pi \sin (\pi z / h)$ & TSDBT \\
$z$ & $-z e^{-2 z^{2} / h^{2}}$ & ESDBT \\
$z$ & $z \operatorname{sech} \pi / 4(1-\pi / 2 \tanh \pi / 4)-z \operatorname{sech} \pi z^{2} / h^{2}$ & HSDBT \\
\hline
\end{tabular}

The axial strain $\left(\varepsilon_{x x}\right)$ and shear strain $\left(\gamma_{x z}\right)$ resulted from eq. (4) are of the form

$$
\varepsilon_{x x}=u_{, x}-g(z) w_{, x x}-f(z) \theta_{, x}, \quad \gamma_{x z}=\left[1-g_{, z}(z)\right] w_{, x}-f_{, z}(z) \theta
$$

The constitutive equations based on linear behaviour of the material are

$$
\sigma_{x x}=E_{f}(x, z) \varepsilon_{x x}, \quad \tau_{x z}=G_{f}(x, z) \gamma_{x z}
$$

where $\sigma_{x x}$ and $\tau_{x z}$ are, respectively, the axial and shear stresses; $G_{f}(x, z)$ is the effective shear modulus and it is defined by eq. (3). 
Transport and Communications Science Journal, Vol. 72, Issue 4 (05/2021), 452-467

The elastic strain energy of the beam $\left(U_{\mathrm{B}}\right)$ is given by

$$
U_{\mathrm{B}}=\frac{1}{2} \int_{0}^{L} \int_{A}\left(\sigma_{x x} \varepsilon_{x x}+\tau_{x z} \gamma_{x z}\right) d A d x
$$

where $A$ is the cross-sectional area of the beam.

Substituting eqs. (5) and (6) into eq. (7), one gets

$$
\begin{aligned}
U_{\mathrm{B}}=\frac{1}{2} \int_{0}^{L}\left[A_{11} u_{, x}^{2}-2 A_{12} u_{, x} w_{, x x}+A_{22} w_{, x x}^{2}-2 A_{13} u_{, x} \theta_{, x}+2 A_{23} w_{, x x} \theta_{, x}+A_{33} \theta_{, x}^{2}\right. \\
\left.+B_{22} w_{, x}^{2}-2 B_{23} w_{, x} \theta+B_{33} \theta^{2}\right] d x
\end{aligned}
$$

where the rigidities $A_{11}, A_{12}, A_{22}, A_{13}, A_{23}, A_{33}, B_{22}, B_{23}$ and $B_{33}$ are defined as follows

$$
\begin{aligned}
& \left(A_{11}, A_{12}, A_{22}, A_{13}, A_{23}, A_{33}\right)(x)=b \int_{z_{0}}^{z_{3}} E_{f}(x, z)\left[1, g(z), g^{2}(z), f(z), f(z) g(z), f^{2}(z)\right] d z \\
& \left(B_{22}, B_{23}, B_{33}\right)(x)=b \int_{z_{0}}^{z_{3}} G_{f}(x, z)\left\{\left[1-g_{, z}(z)\right]^{2},\left[1-g_{, z}(z)\right] f_{, z}(z), f_{, z}^{2}(z)\right\} d z
\end{aligned}
$$

The strain energy stored in the elastic foundation $\left(U_{\mathrm{F}}\right)$ is defined by

$$
U_{\mathrm{F}}=\frac{b}{2} \int_{0}^{L_{F}}\left[k_{w} w^{2}+k_{s} w_{, x}^{2}\right] d x
$$

The kinetic energy $(T)$ of the beam is defined as

$$
T=\frac{1}{2} \int_{0}^{L} \int_{A} \rho_{f}(x, z)\left(\dot{u}_{1}^{2}+\dot{u}_{3}^{2}\right) d A d x
$$

where an over dot is used to denote the derivative with respect to the time variable. From eq. (4), the kinetic energy of the beam can be rewritten in the form

$$
T=\frac{1}{2} \int_{0}^{L}\left[I_{11}\left(\dot{u}^{2}+\dot{w}^{2}\right)-2 I_{12} \dot{u} \dot{w}_{, x}+I_{22} \dot{w}_{, x}^{2}-2 I_{13} \dot{u} \dot{\theta}+2 I_{23} \dot{w}_{, x} \dot{\theta}+I_{33} \dot{\theta}^{2}\right] d x
$$

where $I_{11}, I_{12}, I_{22}, J_{11}, J_{12}, J_{22}$ are the mass moments of the beam, defined as

$$
\left(I_{11}, I_{12}, I_{22}, I_{13}, I_{23}, I_{33}\right)(x)=b \int_{z_{0}}^{z_{3}} \rho_{f}(x, z)\left[1, g(z), g^{2}(z), f(z), g(z) f(z), f^{2}(z)\right] d z
$$

Applying Hamilton's principle to eqs. (8), (10) and (12), we obtain the following equations of motion for the BFGSW beam partially supported by elastic foundation

$$
\begin{aligned}
& -I_{11} \ddot{u}+I_{12} \ddot{w}_{, x}+I_{13} \ddot{\theta}+\left(A_{11} u_{, x}-A_{12} w_{, x x}-A_{13} \theta_{, x}\right)_{, x}=0 \\
& I_{11} \ddot{w}+\left(I_{12} \ddot{u}-I_{22} \ddot{w}_{, x}-I_{23} \ddot{\theta}\right)_{, x}+\left(B_{23} \theta-B_{22} w_{, x}\right)_{, x} \\
& \quad+\left(-A_{12} u_{, x}+A_{22} w_{, x x}+A_{23} \theta_{, x}\right)_{, x x}+\underbrace{b\left(k_{w} w-k_{s} w_{, x x}\right)}_{0 \leq x \leq L_{F}}=0 \\
& I_{13} \ddot{u}-I_{23} \ddot{w}_{, x}-I_{33} \ddot{\theta}-B_{33} \theta+B_{23} w_{, x}+\left(-A_{13} u_{, x}+A_{23} w_{, x x}+A_{33} \theta_{, x}\right)_{, x}=0
\end{aligned}
$$

Since the beam rigidities and mass moments are dependent on $x$, a closed-form solution 
Transport and Communications Science Journal, Vol. 72, Issue 4 (05/2021), 452-467

for eq. (14) is hardly derived. The finite formulation is employed herein to solve eq. (14) and to compute frequencies of the beam.

\section{FINITE ELEMENT FORMULATION}

The beam is assumed to be divided into a number of elements with length $l$. The vector of nodal displacement (d) for two-node beam element contains eight components as

$$
\mathbf{d}=\left\{\begin{array}{lll}
\mathbf{d}_{u} & \mathbf{d}_{w} & \mathbf{d}_{\theta}
\end{array}\right\}^{T}
$$

where

$$
\mathbf{d}_{u}=\left\{\begin{array}{ll}
u_{1} & u_{2}
\end{array}\right\}^{T}, \quad \mathbf{d}_{w}=\left\{\begin{array}{llll}
w_{1} & w_{, x 1} & w_{2} & w_{, x 2}
\end{array}\right\}^{T}, \quad \mathbf{d}_{\theta}=\left\{\begin{array}{ll}
\theta_{1} & \theta_{2}
\end{array}\right\}^{T}
$$

are, respectively, the vectors of the nodal axial, bending and shear displacements; The superscript ' $T$ ' in eq. (16) and hereafter is used to denote the transpose of a vector or a matrix.

The displacements are interpolated from their nodal values according to

$$
u=\mathbf{N d}_{u}, \quad w=\mathbf{H d}_{w}, \quad \theta=\mathbf{N d}_{\theta}
$$

where $\mathbf{N}=\left[\begin{array}{ll}N_{1} & N_{2}\end{array}\right]$ and $\mathbf{H}=\left[\begin{array}{llll}H_{1} & H_{2} & H_{3} & H_{4}\end{array}\right]$ are the matrices of interpolation functions. The following linear and cubic Hermite polynomials are respectively used in the present work

$$
\begin{aligned}
& N_{1}=\frac{l-x}{l}, \quad N_{2}=\frac{x}{l} \\
& H_{1}=1-3 \frac{x^{2}}{l^{2}}+2 \frac{x^{3}}{l^{3}}, \quad H_{2}=x-2 \frac{x^{2}}{l}+\frac{x^{3}}{l^{2}}, \quad H_{3}=3 \frac{x^{2}}{l^{2}}-2 \frac{x^{3}}{l^{3}}, \quad H_{4}=-\frac{x^{2}}{l}+\frac{x^{3}}{l^{2}}
\end{aligned}
$$

Using the interpolations, one can write the strain energy $\left(U_{\mathrm{B}}\right)$ of the beam in the form

$$
U_{\mathrm{B}}=\frac{1}{2} \sum^{N E B} \mathbf{d}^{T} \mathbf{k}_{\mathrm{B}} \mathbf{d}
$$

where $N E B$ is the total number of elements used to discrete the beam; $\mathbf{k}_{\mathrm{B}}$ is the element stiffness matrix which can be written in sub-matrices as

$$
\underset{8 \times 8}{\mathbf{k}_{\mathrm{B}}}=\left[\begin{array}{ccc}
\mathbf{k}_{u u}^{\mathrm{B}} & \mathbf{k}_{u w}^{\mathrm{B}} & \mathbf{k}_{u \theta}^{\mathrm{B}} \\
\left(\mathbf{k}_{u w}^{\mathrm{B}}\right)^{T} & \mathbf{k}_{w w}^{\mathrm{B}} & \mathbf{k}_{w \theta}^{\mathrm{B}} \\
\left(\mathbf{k}_{u \theta}^{\mathrm{B}}\right)^{T} & \left(\mathbf{k}_{w \theta}^{\mathrm{B}}\right)^{T} & \mathbf{k}_{\theta \theta}^{\mathrm{B}}
\end{array}\right]
$$

with $\mathbf{k}_{u u}^{\mathrm{B}}, \mathbf{k}_{w w}^{\mathrm{B}}, \mathbf{k}_{\theta \theta}^{\mathrm{B}}, \mathbf{k}_{u w}^{\mathrm{B}}, \mathbf{k}_{u \theta}^{\mathrm{B}}, \mathbf{k}_{w \theta}^{\mathrm{B}}$ are, respectively, the element stiffness matrices stemming from the axial, bending, shear, axial-bending coupling, axial-shear coupling and bending-shear coupling deformation. The expressions for these matrices are as follows

$$
\begin{aligned}
& \mathbf{k}_{u u}^{\mathrm{B}}=\int_{0}^{l} \mathbf{N}_{, x}^{T} A_{11} \mathbf{N}_{, x} d x, \quad \mathbf{k}_{w w}^{\mathrm{B}}=\int_{0}^{l}\left(\mathbf{H}_{, x x}^{T} A_{22} \mathbf{H}_{, x x}+\mathbf{H}_{, x}^{T} B_{22} \mathbf{H}_{, \mathrm{x}}\right) d x \\
& \mathbf{k}_{\theta \theta}^{\mathrm{B}}=\int_{0}^{l}\left(\mathbf{N}_{, x}^{T} A_{33} \mathbf{N}_{, x}+\mathbf{N}^{T} B_{33} \mathbf{N}\right) d x, \quad \mathbf{k}_{u w}^{\mathrm{B}}=-\int_{0}^{l} \mathbf{N}_{, x}^{T} A_{12} \mathbf{H}_{, x x} d x \\
& \mathbf{k}_{u \theta}^{\mathrm{B}}=-\int_{0}^{l} \mathbf{N}_{, x}^{T} A_{13} \mathbf{N}_{, x} d x, \quad \mathbf{k}_{w \theta}^{\mathrm{B}}=\int_{0}^{l}\left(\mathbf{H}_{, x x}^{T} A_{23} \mathbf{N}_{, \mathrm{x}}-\mathbf{H}_{, x}^{T} B_{23} \mathbf{N}\right) d x
\end{aligned}
$$

The strain energy $\left(U_{\mathrm{F}}\right)$ in eq. (10) can also be written as 
Transport and Communications Science Journal, Vol. 72, Issue 4 (05/2021), 452-467

$$
U_{\mathrm{F}}=\frac{1}{2} \sum^{N E F} \mathbf{d}^{T} \mathbf{k}_{\mathrm{F}} \mathbf{d}
$$

where $N E F$ is the number of elements used for the foundation; $\mathbf{k}_{\mathrm{F}}$ is the element foundation stiffness matrix which can also be written in the following form

$$
\underset{8 \times 8}{\mathbf{k}_{\mathrm{F}}}=\left[\begin{array}{ccc}
0 & 0 & 0 \\
0 & \mathbf{k}_{w w}^{\mathrm{F}} & 0 \\
0 & 0 & 0
\end{array}\right]
$$

where

$$
\mathbf{k}_{w w}^{\mathrm{F}}=b \int_{0}^{l}\left(\mathbf{H}^{T} k_{w} \mathbf{H}+\mathbf{H}_{, x}^{T} k_{s} \mathbf{H}_{, x}\right) d x
$$

The total element stiffness is

$$
\mathbf{k}=\mathbf{k}_{\mathrm{B}}+\mathbf{k}_{\mathrm{F}}
$$

for the element on the foundation and $\mathbf{k}=\mathbf{k}_{\mathrm{B}}$ for the element without the foundation support.

The kinetic energy in eq. (12) can also be written in the form

$$
T=\frac{1}{2} \sum^{N E B} \dot{\mathbf{d}}^{T} \mathbf{m} \dot{\mathbf{d}}
$$

where the element mass matrix $\mathbf{m}$ can be written in sub-matrices as follows

$$
\mathbf{m}_{8 \times 8}=\left[\begin{array}{ccc}
\mathbf{m}_{u u} & \mathbf{m}_{u w} & \mathbf{m}_{u \theta} \\
\left(\mathbf{m}_{u w}\right)^{T} & \mathbf{m}_{w w} & \mathbf{m}_{w \theta} \\
\left(\mathbf{m}_{u \theta}\right)^{T} & \left(\mathbf{m}_{w \theta}\right)^{T} & \mathbf{m}_{\theta \theta}
\end{array}\right]
$$

in which

$$
\begin{aligned}
& \mathbf{m}_{u u}=\int_{0}^{l} \mathbf{N}^{T} I_{11} \mathbf{N} d x, \quad \mathbf{m}_{w w}=\int_{0}^{l}\left(\mathbf{H}^{T} I_{11} \mathbf{H}+\mathbf{H}_{, x}^{T} I_{22} \mathbf{H}_{, \mathrm{x}}\right) d x, \quad \mathbf{m}_{\theta \theta}=\int_{0}^{l} \mathbf{N}^{T} I_{33} \mathbf{N} d x, \\
& \mathbf{m}_{u w}=-\int_{0}^{l} \mathbf{N}^{T} I_{12} \mathbf{H}_{, \mathrm{x}} d x, \quad \mathbf{m}_{u \theta}=-\int_{0}^{l} \mathbf{N}^{T} I_{13} \mathbf{N} d x, \quad \mathbf{m}_{w \theta}=\int_{0}^{l} \mathbf{H}_{, x}^{T} I_{23} \mathbf{N} d x
\end{aligned}
$$

The discrete equation of motion for the beam has the form

$$
\mathbf{M D}+\mathbf{K D}=\mathbf{0}
$$

where $\mathbf{D}, \ddot{\mathbf{D}}$ are the vectors of nodal displacement and acceleration, respectively; $\mathbf{M}$ and $\mathbf{K}$ are, respectively, the global mass and stiffness matrices obtained by assembling the matrices $\mathbf{m}$ and $\mathbf{k}$ over the elements. Assuming a harmonic form for the vector of the nodal displacements, eq. (29) leads to an eigenvalue problem for determining the frequency $\omega$ as

$$
\left(\mathbf{K}-\omega^{2} \mathbf{M}\right) \overline{\mathbf{D}}=\mathbf{0}
$$


Transport and Communications Science Journal, Vol. 72, Issue 4 (05/2021), 452-467

with $\overline{\mathbf{D}}$ is the vibration amplitude. Solution of eq. (30) can be obtained by the standard method.

\section{NUMERICAL INVESTIGATION}

Natural frequencies of the simply-supported BFGSW beam partially resting on a Pasternak foundation are evaluated in this section. To this end, the beam with $h=1 \mathrm{~m}, b=1 \mathrm{~m}$, made from alumina $\left(\mathrm{Al}_{2} \mathrm{O}_{3}\right)$ as $\mathrm{M}_{1}$, zirconia $\left(\mathrm{ZrO}_{2}\right)$ as $\mathrm{M}_{2}$ and aluminum ( $\left.\mathrm{Al}\right)$ as $\mathrm{M}_{3}$ with their material properties stated in table $2[12,18,19]$ is considered.

Table 2. Properties of constituent materials of the BFGSW beam.

\begin{tabular}{lllll}
\hline Materials & Role & $E(\mathrm{GPa})$ & $\rho\left(\mathrm{kg} / \mathrm{m}^{3}\right)$ & $v$ \\
\hline Alumina $\left(\mathrm{Al}_{2} \mathrm{O}_{3}\right)$ & $\mathrm{M}_{1}$ & 380 & 3960 & 0.3 \\
Zirconia $\left(\mathrm{ZrO}_{2}\right)$ & $\mathrm{M}_{2}$ & 151 & 3000 & 0.3 \\
Aluminum $(\mathrm{Al})$ & $\mathrm{M}_{3}$ & 70 & 2702 & 0.3 \\
\hline
\end{tabular}

The following dimensionless parameters are introduced for frequencies and foundation stiffness parameters as [12]

$$
\mu=\omega \frac{L^{2}}{h} \sqrt{\frac{\rho_{\mathrm{Al}}}{E_{\mathrm{Al}}}}, \quad\left(k_{1}, k_{2}\right)=\left(\frac{k_{w} L^{4}}{I E_{\mathrm{Al}_{2} \mathrm{O}_{3}}}, \frac{k_{s} L^{2}}{I E_{\mathrm{Al}_{2} \mathrm{O}_{3}}}\right)
$$

where $\omega$ is the fundamental frequency. The layer thickness ratio is denoted by three numbers, e.g. (2-1-1) means that the thickness ratio of the bottom, core and top layers is 2:1:1.

Table 3. Comparison of frequency parameter of BFGSW beam without foundation.

\begin{tabular}{|c|c|c|c|c|c|c|c|c|c|c|}
\hline \multirow[b]{2}{*}{ Theory } & \multirow[b]{2}{*}{$n_{x}$} & \multirow[b]{2}{*}{$n_{z}$} & \multicolumn{4}{|c|}{$L / h=5$} & \multicolumn{4}{|c|}{$L / h=20$} \\
\hline & & & $2-1-2$ & $2-1-1$ & $1-1-1$ & $2-2-1$ & $2-1-2$ & $2-1-1$ & $1-1-1$ & $2-2-1$ \\
\hline Ref. [6] & 1 & 0.5 & 3.8437 & 3.7805 & 3.7428 & 3.6610 & 4.2429 & 4.1592 & 4.1375 & 4.0333 \\
\hline CBT & & & 4.1133 & 4.0364 & 4.0253 & 3.92 & 4.1798 & 4.1013 & 4.0 & \\
\hline FSDBT & & & 3.8648 & 3.7964 & 3.7794 & 3.6939 & 4.1618 & 4.0840 & 4.0725 & 3.9760 \\
\hline PSDBT & & & 3.7609 & 3.7038 & 3.6746 & 3.6006 & 4.1528 & 4.0760 & 4.0635 & 3.9680 \\
\hline TSDBT & & & & 3.7 & $3.6^{\circ}$ & 3.5 & & 758 & & \\
\hline ESDBT & & & 3.7545 & 3.7006 & 3.6696 & 3.5 & & 4.0757 & & 3.9677 \\
\hline HSD & & & 3.7741 & 3.7130 & 3.68 & 3.60 & & 4.0768 & & 3.9686 \\
\hline Ref & & 2 & 757 & 4.3 & 4.3 & 4.2 & 4. & 33 & 4.8 & 4.7 \\
\hline CBT & & & 2 & 4.6251 & 4.6 & 4.5 & 4.8 & 4.7007 & & \\
\hline & & & 4.4333 & 4.3438 & 4.3599 & 4.2 & 4.7 & 4.6804 & & 4.5880 \\
\hline & & & 4.2956 & 4.2241 & 4.2001 & 4.10 & 4.7697 & 4.6700 & & 4.5757 \\
\hline TSDBT & & & 4.2863 & 4.2186 & 4.1896 & 4.09 & 4.7688 & 4.6695 & & 4.5750 \\
\hline ESD & & & 4.2777 & 4.2144 & 4.1801 & 4.09 & 4.7680 & 4.6691 & & 4.5745 \\
\hline HSDBT & & & & 4.2 & 4.22 & 4.12 & & 4.6715 & & 4.5775 \\
\hline ef. [6] & 5 & 0.5 & 3.3237 & 3.2 & 3.26 & 3.2 & 3.6 & 3.5602 & 3 & 81 \\
\hline CBT & & & 3.5564 & 3.5098 & 3.4989 & 3.4 & & 3.5663 & & 3.4966 \\
\hline FSDBT & & & 3.3786 & 3.3357 & 3.3215 & 3.26 & & 3.5540 & & 3.4843 \\
\hline PSDBT & & & 3.3113 & 3.2742 & 3.2536 & 3.2061 & & 3.5488 & & 3.4791 \\
\hline TSDBT & & & 3.3092 & 3.2730 & 3.2520 & 3.2051 & 3.5954 & 3.5486 & 3.5368 & 3.4789 \\
\hline ESDBT & & & 3.3080 & 3.2726 & 3.2512 & 3.2049 & 3.5952 & 3.5486 & 3.5367 & 3.4789 \\
\hline
\end{tabular}


Transport and Communications Science Journal, Vol. 72, Issue 4 (05/2021), 452-467

\begin{tabular}{|c|c|c|c|c|c|c|c|c|c|}
\hline HSDBT & & 3.3192 & 3.2800 & 3.2604 & 3.2112 & 3.5962 & 3.5492 & 3.5375 & 3.4795 \\
\hline Ref. [6] & 2 & 3.7155 & 3.6618 & 3.6419 & 3.5719 & 4.0422 & 3.9737 & 3.9825 & 3.8972 \\
\hline CBT & & 3.9711 & 3.9049 & 3.9181 & 3.8357 & 4.0369 & 3.9693 & 3.9831 & 3.8990 \\
\hline FSDBT & & 3.7747 & 3.7133 & 3.7166 & 3.6395 & 4.0230 & 3.9557 & 3.9688 & 3.8851 \\
\hline PSDBT & & 3.6895 & 3.6376 & 3.6201 & 3.5525 & 4.0159 & 3.9493 & 3.9606 & 3.8777 \\
\hline TSDBT & & 3.6844 & 3.6345 & 3.6146 & 3.5488 & 4.0154 & 3.9490 & 3.9601 & 3.8774 \\
\hline ESDBT & & 3.6799 & 3.6323 & 3.6099 & 3.5460 & 4.0150 & 3.9488 & 3.9597 & 3.8771 \\
\hline HSDBT & & 3.7053 & 3.6481 & 3.6365 & 3.5644 & 4.0171 & 3.9502 & 3.9620 & 3.8787 \\
\hline
\end{tabular}

Before computing the frequencies, the accuracy of the derived formulation is necessary to verify. To this end, in Table 3 the frequency parameters of the BFGSW beam with $L / h=5$ and $L / h=20$ without foundation support obtained in the present paper are compared with the result of Ref. [6] using the third-order shear deformation beam theory. It is seen from Table 3 that the frequency parameters based on the shear deformation theories (FSDBT, TSDBT, ESDBT, HSDBT) are close to result of Ref. [6], while difference between the present frequencies based on CBT with that of Ref. [6] is significant for $L / h=5$, regardless of the layer thickness ratio and the grading index. This difference can be explained by the fact that the CBT does not consider the warping of the cross-section of the beam after bending. For the slender beam, as $L / h=20$, the difference is negligible.

Table 4 compares the frequency parameters of a unidirectional FGSW beam fully rested on the Pasternak foundation of the present work with the result of Su et al. [12] using the FSDBT and the general Fourier formulation. The sandwich beam in [12] is a special case of the present beam when $n_{x}=0$. As seen from Table 4 , the result obtained from all above beam theories except the CBT in this paper are in excellent agreement with that of Ref. [12], regardless of the grading index $n_{z}$ and the foundation stiffness. For $L / h=10$, the difference between the result obtained from CBT and Ref. [12] is very relatively small.

Table 4. Comparison of frequency parameters of (1-1-1) FGSW beam fully resting on foundation

\begin{tabular}{|c|c|c|c|c|c|c|}
\hline \multirow[t]{2}{*}{ Theory } & \multirow[t]{2}{*}{$\mathrm{k}_{1}$} & \multirow{2}{*}{$\frac{k_{2}}{\pi^{2}}$} & \multicolumn{4}{|c|}{$n_{z}$} \\
\hline & & & 0.6 & 1 & 2 & 5 \\
\hline Ref. [12] & 0 & 0.5 & 5.9427 & 5.7192 & 5.4540 & 5.2632 \\
\hline CBT & & & 5.9715 & 5.7382 & 5.4658 & 5.2704 \\
\hline FSDBT & & & 5.9416 & 5.7152 & 5.4502 & 5.2597 \\
\hline PSDBT & & & 5.9384 & 5.7135 & 5.4498 & 5.2601 \\
\hline TSDBT & & & 5.9384 & 5.7135 & 5.4498 & 5.2602 \\
\hline ESDBT & & & 5.9386 & 5.7136 & 5.4500 & 5.2604 \\
\hline HSDBT & & & 5.9385 & 5.7135 & 5.4496 & 5.2598 \\
\hline Ref. [12] & & 1 & 7.1784 & 7.0289 & 6.8652 & 6.7699 \\
\hline CBT & & & 7.2017 & 7.0440 & 6.8743 & 6.7752 \\
\hline FSDBT & & & 7.1772 & 7.0256 & 6.8621 & 6.7671 \\
\hline PSDBT & & & 7.1746 & 7.0242 & 6.8618 & 6.7674 \\
\hline TSDBT & & & 7.1746 & 7.0242 & 6.8618 & 6.7675 \\
\hline ESDBT & & & 7.1747 & 7.0243 & 6.8619 & 6.7676 \\
\hline
\end{tabular}


Transport and Communications Science Journal, Vol. 72, Issue 4 (05/2021), 452-467

\begin{tabular}{|c|c|c|c|c|c|c|}
\hline HSDBT & & & 7.1747 & 7.0242 & 6.8617 & 6.7672 \\
\hline Ref. [12] & 100 & 0.5 & 8.2828 & 8.1846 & 8.0894 & 8.0576 \\
\hline CBT & & & 8.3025 & 8.1974 & 8.0969 & 8.0619 \\
\hline FSDBT & & & 8.2816 & 8.1819 & 8.0868 & 8.0553 \\
\hline PSDBT & & & 8.2793 & 8.1806 & 8.0865 & 8.0555 \\
\hline TSDBT & & & 8.2793 & 8.1806 & 8.0866 & 8.0556 \\
\hline ESDBT & & & 8.2794 & 8.1807 & 8.0866 & 8.0557 \\
\hline HSDBT & & & 8.2794 & 8.1807 & 8.0865 & 8.0553 \\
\hline Ref. [12] & & 1 & 9.2097 & 9.1479 & 9.1007 & 9.1134 \\
\hline CBT & & & 9.2270 & 9.1591 & 9.1072 & 9.1170 \\
\hline FSDBT & & & 9.2084 & 9.1454 & 9.0984 & 9.1113 \\
\hline PSDBT & & & 9.2064 & 9.1443 & 9.0981 & 9.1115 \\
\hline TSDBT & & & 9.2064 & 9.1443 & 9.0981 & 9.1116 \\
\hline ESDBT & & & 9.2065 & 9.1444 & 9.0982 & 9.1117 \\
\hline HSDBT & & & 9.2065 & 9.1443 & 9.0980 & 9.1114 \\
\hline
\end{tabular}

The fundamental frequency parameters of the BFGSW beam with $L / h=5$ and $L / h=20$ obtained by the different beam theories are presented in Tables 5 and 6 for various values of the layer thickness ratio, material grading indexes and foundation supporting parameter. Observation from these tables shows that the frequency parameters based on the CBT are slightly higher than the ones calculated from the other shear deformation beam theories. The difference between the results obtained from the CBT and the shear deformation beam theories decreases as the value of $L / h$ increases, regardless of the considered parameters. However, the difference between the frequency parameters obtained from the shear deformation beam theories is not signification. The layer thickness ratio, material grading indexes and foundation supporting parameter $\alpha_{F}$ have signification influence on the frequency parameter of the beam. As seen from Tables 5 and 6, the frequency parameter is higher for the beam associated with a larger core thickness and a higher parameter $\alpha_{F}$, regardless of the aspect ratio, material grading indexes and beam theories. In addition, the frequency parameter increases with the increase of the index $n_{x}$ and the decrease of $n_{z}$, regardless of layer thickness ratio, the foundation supporting parameter and aspect ratio as well. The dependence of the frequency parameter on the grading indexes can be explained by the change in the constituent volume fraction as seen from eq. (1).

Table 5. Frequency parameters of BFGSW beam partially resting on elastic foundation with $\left(k_{1}, k_{2}\right)=(100,10), L / h=5$.

\begin{tabular}{|c|c|c|c|c|c|c|c|c|c|c|}
\hline \multirow[b]{2}{*}{ Theories } & \multirow[b]{2}{*}{$n_{x}$} & \multirow[b]{2}{*}{$n_{z}$} & \multicolumn{4}{|c|}{$\alpha_{F}=0.4$} & \multicolumn{4}{|l|}{$\alpha_{F}=0.8$} \\
\hline & & & $2-1-2$ & $2-1-1$ & $1-1-1$ & $2-2-1$ & $2-1-2$ & $2-1-1$ & $1-1-1$ & $2-2-1$ \\
\hline CBT & 0.5 & 1 & 6.2783 & 6.3542 & 6.3460 & 6.4319 & 8.2144 & 8.2568 & 8.2246 & 8.2708 \\
\hline FSDBT & & & 6.1375 & 6.2085 & 6.2045 & 6.28 & 8.1146 & 8.1524 & 8.1 & 8.1639 \\
\hline PSDBT & & & 1307 & 6. & 6.1 & 6.2 & 05 & 8.1 & 8 & 8. \\
\hline TSD & & & 17 & 6.1983 & 6.1 & 6.2 & 8.1113 & 8.1459 & 8. & 8.1553 \\
\hline ESDBT & & & 6.1330 & 6.1993 & 6.1964 & 6.2725 & 8.1123 & 8.1466 & 8.1 & 8.1559 \\
\hline HSDBT & & & 6.1294 & 6.1977 & 6.19 & 6.27 & 95 & 8.1453 & 67 & 8.1554 \\
\hline CBT & & 5 & 5.8019 & 5.9330 & 5.8558 & 6.0031 & 8.0680 & 8.1303 & 8.0219 & 8.0897 \\
\hline
\end{tabular}


Transport and Communications Science Journal, Vol. 72, Issue 4 (05/2021), 452-467

\begin{tabular}{|c|c|c|c|c|c|c|c|c|c|c|}
\hline FSDBT & & & 5.6643 & 5.7923 & 5.7307 & 5.8736 & 7.9789 & 8.0366 & 7.9387 & 8.0010 \\
\hline PSDBT & & & 5.6757 & 5.7946 & 5.7368 & 5.8728 & 7.9871 & 8.0386 & 7.9433 & 8.0009 \\
\hline TSDBT & & & 6788 & 5.7963 & 5.7386 & 5.8739 & 7.9891 & 8.0398 & 7.9445 & 8.0017 \\
\hline ESDBT & & & 6820 & 5.7983 & 5.7407 & 5.8754 & 12 & 8.0 & 7.9459 & 8.0027 \\
\hline HSDBT & & & .6678 & 5.7913 & 5.7328 & $5.8^{\prime}$ & & & 7.9407 & 7.9997 \\
\hline CBT & 2 & 1 & 5 & 6. & 51 & 7 & 50 & 22 & 2826 & 3231 \\
\hline FSDBT & & & 3 & 6.3 & & & & & 22 & 8.2080 \\
\hline PSDBT & & & 6.2415 & 6.2980 & 6.2932 & 6.3 & 13 & 8. & 8.1656 & 8.1985 \\
\hline TSDBT & & & .2426 & 6.2987 & 6.2939 & 6.3 & 22 & 8. & 62 & 8.1989 \\
\hline ESDBT & & & 6.2441 & 6.2 & 6.2950 & 6.3 & & & 70 & 8.1996 \\
\hline HSDBT & & & 6.2400 & 6.2978 & 6.2931 & & & & 8.1654 & 8.1988 \\
\hline CBT & & 5 & 0332 & 61 & .0620 & & & & 130 & 8.1708 \\
\hline FSDBT & & & 5.8847 & 5.9868 & 5.9252 & & & 66 & 8.0 & 8.0716 \\
\hline PSDBT & & & 5.8940 & 5.9872 & 5.9305 & & & & 8.0240 & 8.0715 \\
\hline TSDBT & & & .8971 & 5.9891 & 5.9326 & 6.0401 & 8.0685 & 8.1097 & 8.0255 & 8.0725 \\
\hline ESDBT & & & 5.9006 & 5.9913 & 5.9349 & 6.0418 & 8.0709 & 8.1112 & 8.0270 & 8.0736 \\
\hline HSDBT & & & 5.8858 & 5.9837 & 5.9259 & 6.0365 & 8.0606 & 8.1059 & 8.0208 & 8.0699 \\
\hline
\end{tabular}

The effects of the transverse grading index $n_{z}$ on the fundamental frequency parameter of (2-1-2) beam with $L / h=5$ and $L / h=10$ by using the beam theories are detailed in Fig. 3 . The curves in the figure show that the frequency parameter decreases by increasing $n_{z}$, regardless of the aspect ratio and the beam theory. There is a more significant difference between the frequency parameter obtained from the CBT and the shear deformation theories when the value of $L / h$ is smaller. Since the frequency curves obtained from the shear deformation beam theories are very close to each other, the HSDBT is chosen to plot the figures below.

Table 6. Frequency parameters of BFGSW beam partially resting on elastic foundation with $\left(k_{1}, k_{2}\right)=(100,10), L / h=20$.

\begin{tabular}{|c|c|c|c|c|c|c|c|c|c|c|}
\hline \multirow[b]{2}{*}{ Theory } & \multirow[b]{2}{*}{$n_{x}$} & \multirow[b]{2}{*}{$n_{z}$} & \multicolumn{4}{|l|}{$\alpha_{F}=0.4$} & \multicolumn{4}{|l|}{$\alpha_{F}=0.8$} \\
\hline & & & $2-1-2$ & $2-1-1$ & $1-1-1$ & $2-2-1$ & $2-1-2$ & $2-1-1$ & $1-1-1$ & $2-2-1$ \\
\hline CBT & 0.5 & 1 & 6.3683 & 6.4458 & 6.4364 & 6.524 & 8.3306 & 8.3744 & 8.3405 & 8.3883 \\
\hline FSDBT & & & 6.3588 & 6.4358 & 6.4268 & 6.5140 & 3234 & 8.3667 & 8.3331 & 8.3804 \\
\hline PSDBT & & & 580 & 6.4347 & 6.4258 & & 3228 & 8.3659 & 8.3323 & 8.3794 \\
\hline TSDBT & & & 6.3579 & 6.4347 & 6.4257 & 6.51 & .3227 & 8.3659 & 8.3323 & 8.3793 \\
\hline ESDBT & & & 6.3580 & 6.4347 & 6.4258 & 6.5 & 8.3228 & 8.3659 & 8.3323 & 8.3794 \\
\hline HSDBT & & & 78 & 6.4 & 6.4 & & 226 & 8.3659 & 23 & 8.3794 \\
\hline CBT & & 5 & 88 & 6.0223 & 5.9405 & 6.0 & 1850 & 8.2491 & 347 & 8.2042 \\
\hline FSDBT & & & 798 & 6.0126 & 5.9322 & 6.0 & 1785 & 8.2420 & 8.1288 & 8.1976 \\
\hline PSD & & & 5.8802 & 6.0124 & 5.9323 & 6.0 & 8.1789 & 8.2418 & 8.1289 & 8.1973 \\
\hline TSD & & & 03 & 6.0 & 5.9 & & 789 & 8.2418 & 89 & 8.1973 \\
\hline ESL & & & 305 & 6.0 & 5.9324 & & 791 & 8.2419 & 290 & 8.1973 \\
\hline HSDBT & & & 795 & 6.0121 & 5.9319 & 6.0 & 8.1784 & 8.2416 & 8.1286 & 8.1971 \\
\hline CBT & 2 & 1 & 6.4920 & 6.5586 & 6.5471 & 6.6 & 8.3921 & 8.4306 & 995 & 8.4414 \\
\hline FSDB & & & 6.4817 & & 6.5367 & & 8.3840 & 8.4222 & 8.3914 & 8.4329 \\
\hline PSDBT & & & 6.4808 & 6.5466 & 6.5356 & 6.61 & 8.3834 & 8.4213 & 8.3906 & 8.4319 \\
\hline & & & 808 & 6.5 & 6.5356 & 6.6 & 8.3834 & 8.4213 & 8.3905 & 8.4318 \\
\hline ESDBT & & & 6.4809 & 6.5466 & 6.5356 & 6.6102 & 8.3835 & 8.4214 & 8.3906 & 8.4319 \\
\hline
\end{tabular}


Transport and Communications Science Journal, Vol. 72, Issue 4 (05/2021), 452-467

\begin{tabular}{llllllllll} 
HSDBT & & 6.4806 & 6.5465 & 6.5355 & 6.6102 & 8.3833 & 8.4213 & 8.3905 & 8.4319 \\
CBT & 5 & 6.1216 & 6.2293 & 6.1485 & 6.2702 & 8.2756 & 8.3303 & 8.2266 & 8.2860 \\
FSDBT & & 6.1117 & 6.2188 & 6.1394 & 6.2605 & 8.2682 & 8.3223 & 8.2198 & 8.2786 \\
PSDBT & & 6.1120 & 6.2185 & 6.1394 & 6.2601 & 8.2685 & 8.3221 & 8.2199 & 8.2783 \\
TSDBT & & 6.1121 & 6.2185 & 6.1394 & 6.2601 & 8.2686 & 8.3222 & 8.2199 & 8.2783 \\
ESDBT & & 6.1123 & 6.2186 & 6.1396 & 6.2602 & 8.2688 & 8.3223 & 8.2200 & 8.2784 \\
HSDBT & & 6.1113 & 6.2182 & 6.1390 & 6.2598 & 8.2680 & 8.3219 & 8.2196 & 8.2781 \\
\hline
\end{tabular}
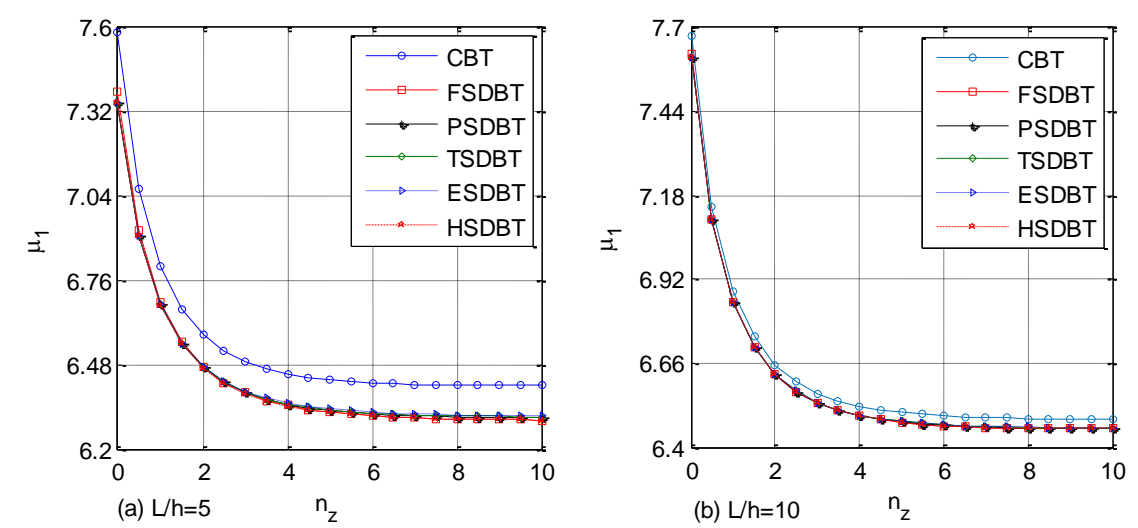

Figure 3. Variation of frequency parameters of (2-1-2) beam with transverse grading index $n_{z}$ for

$$
n_{x}=0.5, \alpha_{F}=0.5,\left(k_{1}, k_{2}\right)=(100,10) \text {. }
$$

The influence of the foundation supporting parameter $\alpha_{F}$ on the relation between the fundamental frequency parameter and the grading indexes $n_{x}$ and $n_{z}$ of (2-1-2) beam obtained by the CBT and HSDBT is displayed in Fig. 4 for various values of $\alpha_{F}$. It is observed from Fig. 4 that the foundation supporting parameter $\alpha_{F}$ has a significant influence on the variation of the frequency parameter with $n_{x}$ and $n_{z}$. The frequency parameter increases by increasing $\alpha_{F}$, regardless of the grading indexes and the beam theory.

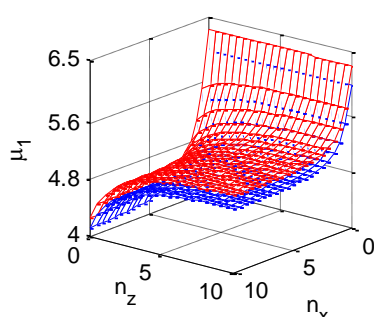

(a) $\alpha_{F}=0.2$

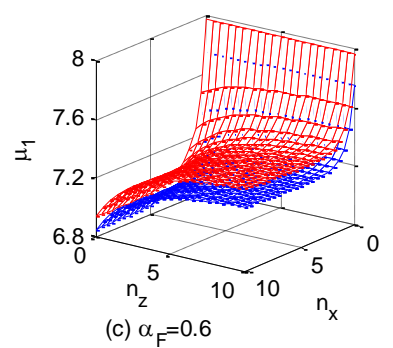

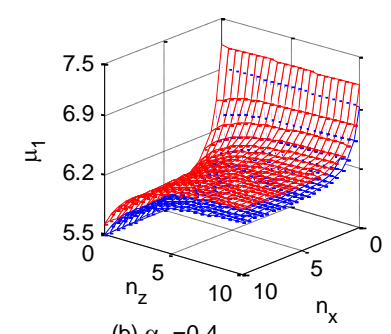

(b) $\alpha_{\mathrm{F}}=0.4$

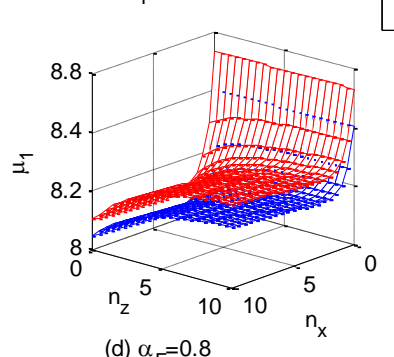

Figure 4. Variation of frequency parameter of (2-1-2) beam with the grading indexes for $\alpha_{F}=0.5,\left(k_{1}\right.$, $\left.k_{2}\right)=(100,10), L / h=5$ and various foundation supporting parameters.

The effect of the foundation stiffness parameters $k_{1}$ and $k_{2}$ on the fundamental frequency 
parameter of (2-1-2) beam based on the CBT and HSDBT is depicted in Fig. 5. The figure shows an increase of the frequency parameter with the increase of parameters $k_{1}$ and $k_{2}$, regardless of the beam theory.

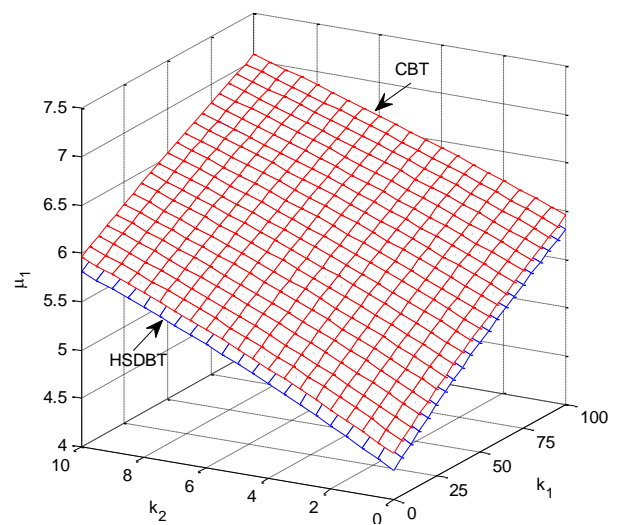

Figure 5. Variation of frequency parameter of (2-1-2) beam with foundation stiffness parameters $k_{l}$ and $k_{2}\left(L / h=5, \alpha_{F}=0.5, n_{x}=n_{z}=0.5\right)$.

For completeness, the effect of the aspect ratio $L / h$ on the frequency parameters of symmetrical (2-1-2) and non-symmetrical (2-2-1) beams is illustrated in Fig. 6 for two pairs of the grading indexes, namely $\left(n_{x}, n_{z}\right)=(0.5,0.5),(3,3)$. As seen from Fig. 6 , the difference between the frequency parameters obtained from the CBT and the HSDBT are significant when the value of the aspect ratio $L / h$ is less than 20 , regardless of the material grading indexes and the beam type. The frequency parameters obtained from the two beam theories are very close to each other when $L / h$ is greater than 20 . Thus, the influence of the shear deformation is significant for the short beam, and in this case the shear deformation beam theories should be employed in the analysis for accounting the shear deformation effect.
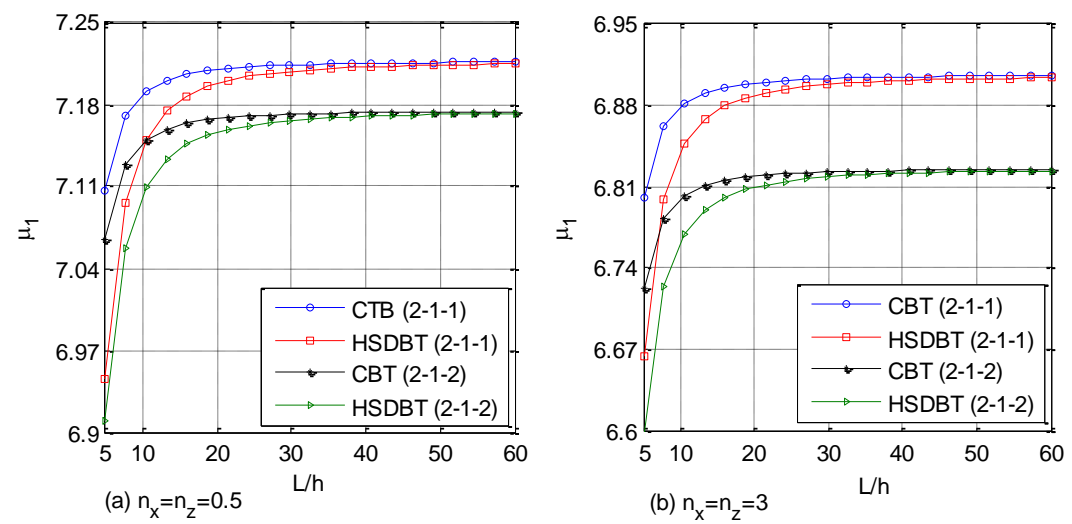

Figure 6. Variation of frequency parameter with aspect ratio for $\left(k_{1}, k_{2}\right)=(100,10), \alpha_{F}=0.5$.

\section{CONCLUSIONS}

Various beam theories have been employed to evaluate the fundamental frequency of the simply supported BFGSW beam partially supported by a Pasternak elastic foundation. The beam with a homogenous core and two FGM face layers is made from three distinct materials. The material properties of the face sheets are considered to vary in both the length and 
thickness directions by the power gradation laws. From the Hamilton's principle, the equations of motion are derived and solved by the finite element method. The accuracy of the derived formulation is confirmed through a comparison study. The numerical investigation obtained in the present paper reveals that the difference between the frequency parameter obtained from the CBT and the shear deformation beam theories is more pronounced for the beam having a smaller the aspect ratio. For the beam with a small aspect ratio, namely $L / h<15$, the shear deformation theory should be employed in evaluating the frequencies to account for the shear deformation effect. The effects of various parameters, including the foundation stiffness, foundation supporting parameter, the material indexes and the layer thickness ratio on the fundamental frequencies of the BFGSW beam have also been studied in detail. It is worthy to note that though the numerical results of the present paper are shown for the beam with simply supported ends only, the formulation derived herein can be used in evaluating frequencies of the BFGSW beams with other boundary conditions as well.

\section{ACKNOWLEDGMENTS}

This research is funded by University of Transport and Communications (UTC) under the project code T2021-CB-009.

\section{REFERENCES}

[1] M. Koizumi, FGM activities in Japan, Composites part B, 28 (1997) 1-4. https://doi.org/10.1016/S1359-8368(96)00016-9

[2] N. A. Apetre, B. V. Sankar, D. R. Ambur, Analytical modeling of sandwich beams with functionally graded core, Journal of sandwich structures and materials, 10 (2008) 53-74. https://doi.org/10.1177/1099636207081111

[3] M. C. Amirani, S. M. R. Khalili, N. Nemati, Free vibration analysis of sandwich beam with FG core using the element free Galerkin method, Composite structures, 90 (2009) 373-379. https://doi.org/10.1016/j.compstruct.2009.03.023

[4] T. P. Vo et al., Finite element model for vibration and buckling of functionally graded sandwich beams based on a refined shear deformation theory, Engineering structures, 64 (2014) 12-22. https://doi.org/10.2016/j.engstruct.2014.01.029

[5] A. Karamanli, Bending behaviour of two directional functionally graded sandwich beams by using a quasi-3d shear deformation theory, Composite structures, 174 (2017) 70-86. https://doi.org/10.1016/j.compstruct.2017.04.046

[6] V. T. A. Ninh, L. T. N. Anh, N. D. Kien, Free vibration of a 2D-FGSW beam based on a shear deformation theory, Vietnam journal of mechanics, VAST, 42 (2020) 189205. https://doi.org/10.15625/0866-7136/14817

[7] D. K. Nguyen et al., Dynamic behavior of a bidirectional functionally graded sandwich beam under nonuniform motion of a moving load, Shock and vibration, 2020 (2020) 15. https://doi.org/10.1155/2020/8854076

[8] M. Eisenberger, D. Z. Yankelevsky, M. A. Adin, Vibrations of beams fully or partially supported on elastic foundations, Earthquake engineering and structural dynamics, 13 (1985) 651-660. https://doi.org/10.1002/eqe.4290130507

[9] W. Q. Chen, C. F. Lu, Z. G. Bian, A mixed method for bending and free vibration of beams resting on a Pasternak elastic foundation, Applied mathematical modelling, 28 (2004) 877-890. https://doi.org/10.1016/j.apm.2004.04.001 
[10] A. M. Zenkour, M. N. M. Allam, M. Sobhy, Bending analysis of FG viscoelastic sandwich beams with elastic cores resting on Pasternak's elastic foundations, Acta mech, 212 (2010) 233-252. https://doi.org/10.1007/s00707-009-0252-6

[11] P. Tossapanon, N. Wattanasakulpong, Stability and free vibration of functionally graded sandwich beams resting on two-parameter elastic foundation, Composite structures, 142 (2016) 215225. https://doi.org/10.1016/j.compstruct.2016.01.085

[12] Z. Su et al., A general Fourier formulation for vibration analysis of functionally graded sandwich beams with arbitrary boundary condition and resting on elastic foundations, Acta mech, 227 (2016) 1493-1514. https://doi.org/10.1007/s00707-016-1575-8

[13] A. S. Sayyad, Y. M. Ghugal, On the free vibration analysis of laminated composite and sandwich plates: A review of recent literature with some numerical results, Composite structures, 129 (2015) 177-201. https://doi.org/10.1016/j.compstruct.2015.04.007

[14] M. Aydogdu, V. Taskin, Free vibration analysis of functionally graded beams with simply supported edges, Materials and design, 28 (2007) 1651-1656. https://doi.org/10.1016/j.matdes.2006.02.007

[15] M. Şimșek, Vibration analysis of a functionally graded beam under a moving mass by using

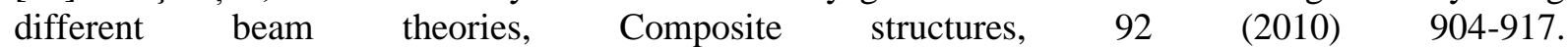
https://doi.org/10.1016/j.compstruct.2009.09.030

[16] A. Mahi et al., An analytical method for temperature-dependent free vibration analysis of functionally graded beams with general boundary conditions, Composite structures, 92 (2010) 1877 1887. https://doi.org/10.1016/j.compstruct.2010.01.010

[17] H-T. Thai, T. P. Vo, Bending and free vibration of functionally graded beams using various higher-order shear deformation beam theories, International journal of mechanical sciences, 62 (2012) 57-66. https://doi.org/10.1016/j.ijmecsci.2012.05.014

[18] M. Şimșek, Non-linear vibration analysis of a functionally graded Timoshenko beam under action $\begin{array}{llllll}\text { of a moving harmonic load, Composite Structures, } 92 & \text { (2010) 2532-2546. }\end{array}$ https://doi.org/10.1016/j.compstruct.2010.02.008

[19] G. N. Praveen, J. N. Reddy, Nonlinear transient thermoelastic analysis of functionally graded ceramic-metal plates, International journal of solids and structures, 35 (1998) 4457-4476. https://doi.org/10.1016/s0020-7683(97)00253-9 